\title{
Belgeo
}

Revue belge de géographie

$1-2 \mid 2010$

Villes et grands équipements de transport/Cities and large transport infrastructures

\section{Baptiser un grand équipement urbain : pratiques et enjeux autour du nom des stations de métro à Bruxelles}

Naming a large urban facility: practices and stakes regarding underground stations in Brussels

\section{Frédéric Dobruszkes}

\section{OpenEdition}

\section{Journals}

\section{Édition électronique}

URL : http://journals.openedition.org/belgeo/7103

DOl : 10.4000/belgeo.7103

ISSN : 2294-9135

Éditeur:

National Committee of Geography of Belgium, Société Royale Belge de Géographie

\section{Édition imprimée}

Date de publication : 30 septembre 2010

Pagination : 229-240

ISSN : 1377-2368

\section{Référence électronique}

Frédéric Dobruszkes, « Baptiser un grand équipement urbain : pratiques et enjeux autour du nom des stations de métro à Bruxelles », Belgeo [En ligne], 1-2 | 2010, mis en ligne le 05 décembre 2012, consulté le 01 mai 2019. URL : http://journals.openedition.org/belgeo/7103 ; DOI : 10.4000/ belgeo.7103

Ce document a été généré automatiquement le 1 mai 2019.

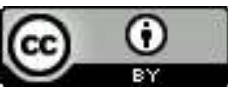

Belgeo est mis à disposition selon les termes de la licence Creative Commons Attribution 4.0 International. 


\title{
Baptiser un grand équipement urbain : pratiques et enjeux autour du nom des stations de métro à Bruxelles
}

\author{
Naming a large urban facility: practices and stakes regarding underground \\ stations in Brussels
}

Frédéric Dobruszkes

\section{Introduction : donner un nom aux lieux, un acte peu anodin}

Depuis que le choix des toponymes relève de décisions étatiques et non plus d'usages populaires préexistants, "nommer le territoire est un acte politique fondateur; le renommer est tout aussi politiquement signifiant" (Giraut et al., 2008). Ceci vaut à toutes les échelles géographiques, depuis le nom des pays jusqu'à celui des rues en passant par celui des villes, que ce soit au moment de leur première attribution toponymique ou à l'occasion de l'attribution d'un nouveau nom (par exemple à la suite de conquêtes, de la (dé)colonisation, de la chute de régimes, etc.). Baptiser les lieux relève de l'action de certains acteurs porteurs, consciemment ou non, de valeurs, de projets (sociaux, économiques, politiques) et de représentations (Guillorel, 2009). La toponymie apparaît ainsi souvent comme l'expression des valeurs des groupes au pouvoir.

2 Ainsi, pour prendre quelques exemples révélateurs, l'urbanisation des nouvelles communes de Barcelone au début du $\mathrm{XX}^{\mathrm{e}}$ siècle est l'occasion d'une glorification systématique de l'histoire catalane: l'homme célébré devient alors un artiste ou un notable catalan plutôt qu'un militaire ou autre royaliste espagnol (Michonneau, 2001). À Naples, dans la seconde moitié du XIX'e, on oscille entre l'exaltation de l'identité locale ou de l'unité nationale, d'une part, et entre choix pittoresques ou folklorisants et réalisme 
social, d'autre part (Broccolini, 2001). Étudiant le nom des rues dans deux villes israéliennes et deux colonies des territoires conquis en 1967, Pinchevski et Torgovnik (2002) montrent notamment que les nouveaux noms participent à la construction d'une mémoire collective (commémoration des héros, batailles, guerres et actions héroïques ayant participé à la création de l'État) remplaçant l'ordre culturel arabe préétabli ; ce recours à une toponymie historiquement connotée peut être plus marqué dans les lieux destinés à accueillir de nombreux immigrants, concourant ainsi à leur intégration sociale et politique à la nation par initiation à la mémoire collective officielle. À Neuilly-surSeine, les rues mettent à l'honneur de nombreux réactionnaires ${ }^{1}$ mais on n'y trouve aucune trace du célèbre résistant communiste Guy Môquet, fusillé en 1941, porteur de quatre décorations officielles et récemment récupéré par le président français (Basse, 2007, cité par Giraut et al., 2008). D'ailleurs, les 44 rues Guy Môquet d'Île-de-France se concentrent surtout dans les bastions communistes, anciens ou persistants ${ }^{2}$. Comme le rappellent Bouvier et Guillon (2001), "la toponymie a ses exclus, parfois ses exécrés, souvent ses vaincus". Outre les personnages n'entrant pas dans les valeurs du moment, les femmes sont significativement peu présentes par rapport aux hommes, par exemple $3 \%$ des noms de rues relatifs à une personne dans le cas de Marseille (Richard, 2001).

3 En plus d'avoir une fonction symbolique et mémorielle, la toponymie a bien sûr également une fonction pratique (Di Méo et Bulion, 2005). De ce point de vue, nommer des stations de métro est un cas particulier d'acte toponymique. En effet, il y a normalement surtout lieu d'aider au repérage des usagers. Circulant souvent sous terre, ces derniers n'ont en effet pas la conscience des territoires traversés comme cela peut être plus facilement le cas avec des trams ou bus circulant en voirie. Le métro se surimposant généralement à un tissu urbain préexistant, il faut souvent faire coïncider le nom des stations avec la toponymie préexistante. La fonction pratique l'emporte donc $a$ priori. Cependant, l'acte toponymique n'est pas pour autant neutre car, dans bien des cas, plusieurs noms peuvent parfaitement s'imposer pour un même lieu, et plus encore si les stations sont dotées d'accès aux deux extrémités des quais comme c'est le cas à Bruxelles (plutôt qu'au niveau d'un seul carrefour comme c'est souvent le cas à Paris). Il n'est donc pas impossible que le nom des stations de métro véhicule certaines valeurs "officielles". Musset (à paraître) montre ainsi qu'à Mexico, le nom des stations "force le voyageur à se replonger dans une histoire officielle et consensuelle chargée de donner du sens à une Nation multiculturelle qui cherche encore son identité entre un passé mal assumé et un présent conflictuel. C'est pourquoi, dans cette longue litanie des grands hommes (et quelques femmes) à qui la Patrie a voulu exprimer sa reconnaissance, les oublis et les absences sont tout aussi révélateurs que les présences." Par ailleurs, comme nous tenterons de le montrer dans le présent article, divers quartiers ne sont pas dotés d'une "identité toponymique" forte, tandis que dans d'autres cas les décideurs se sont écartés des noms les plus logiques ou les plus ancrés dans les pratiques et vécu collectifs. Bien que faisant largement partie du "paysage" et du vécu urbains, le nom des stations de métro ne va donc pas nécessairement de soi.

4 Au travers du cas bruxellois, l'objectif du présent article est d'analyser le processus de décision conduisant à l'attribution d'un nom aux stations du métro par le prisme du mécanisme objectif (procédure officiellement de mise) et des valeurs qui s'expriment à cette occasion dans le chef des responsables publics concernés ${ }^{3}$. Nous tenterons pour cela de nous inscrire dans le courant actuel de recherches toponymiques dites critiques en ce sens qu'elles analysent les jeux de pouvoir inhérents au fait de nommer les lieux plutôt 
que de se contenter de monographies (Vuolteenaho et Berg, 2009). Cette tendance récente est illustrée par Rose-Redwood et al. (à paraître), Berg et Vuolteenaho (2009), un numéro spécial de l'Espace politique ${ }^{4}$ et les auteurs déjà cités.

Pour répondre à cet objectif, nous avons principalement bénéficié de longs et précieux entretiens avec les principaux responsables techniques et administratifs de la construction du métro bruxellois et/ou de la politique des déplacements ${ }^{5}$. Nous avons en outre consulté des cartes topographiques anciennes et, si nécessaire, les plans du métro au 1/500 mis à notre disposition par le ministère.

La suite du texte est composée comme suit. La section suivante explicite la procédure normale d'attribution d'un nom aux stations et les règles officieuses en la matière. Nous détaillerons ensuite leur application et les conséquences sur le nom des stations, avant de nous intéresser aux écarts aux règles et aux enjeux.

\section{De la proposition à la décision}

\section{Un processus émanant des ingénieurs et filtré par le monde politique}

7 La STIB (Société des Transports Intercommunaux de Bruxelles) exploite un réseau de 40 km de métro et 12 km de "pré-métro" (tramways souterrains), doté de 69 stations (un nom = une station) (figure 1). À l'exception de la station Lemonnier aménagée dès les années 1950, ce réseau a été construit entre 1965 et 2009.

Figure 1. Plan du métro bruxellois.

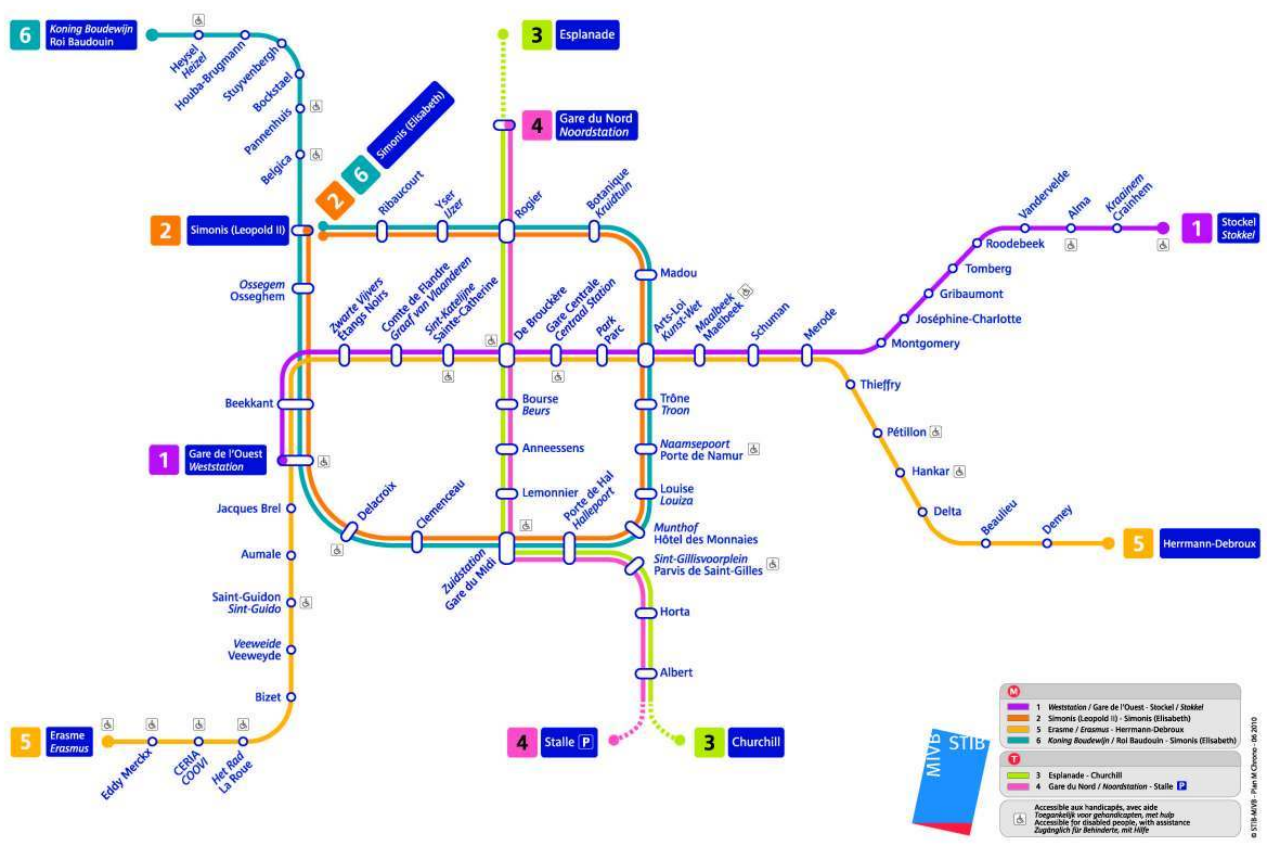

La procédure d'attribution d'un nom à ces stations est somme toute très simple. Dans un premier temps, la STIB propose un nom. Ensuite, le ministre - autrefois national, aujourd'hui régional - ayant la tutelle de la STIB décide. S'il le souhaite, le ministre peut 
demander l'avis de la Direction de l'infrastructure des transports publics de l'administration régionale Bruxelles Mobilité sur la proposition de la STIB.

9 Pour faire simple, les ingénieurs proposent et le ministre dispose. Dans la pratique, ce dernier suit très souvent la proposition initiale. Il faut également signaler que, bien souvent, le nom de la station semble soumis à l'approbation des communes, échelon de pouvoir qui demeure incontournable à Bruxelles malgré l'existence d'une Région depuis 1989. Par contre, la Commission de toponymie et de dialectologie n'est pas consultée, bien que celle-ci se tienne à la disposition des pouvoirs publics en cas de besoin ${ }^{6}$.

10 C'est donc d'un groupe précis qu'émanent de nombreux noms de stations: celui des ingénieurs, naturellement très représentés dans les services techniques et, plus généralement, dans les administrations ou compagnies en charge des transports collectifs.

\section{Des règles non-écrites}

11 Les concepteurs du métro bruxellois se sont dotés de règles officieuses, ou plutôt de guides, pour proposer des noms aux stations.

Tout d'abord, à cause d'une contrainte technique liée aux afficheurs électroniques de destinations, les noms doivent pour bien faire être relativement courts, en particulier pour les terminus tant temporaires que définitifs ainsi que pour les services partiels. Les afficheurs initiaux étant limités à 13 caractères, dont deux pour le numéro de ligne et un espace, il reste donc 10 caractères. Ensuite, on recourt à des noms propres et, si cela s'y prête, à des personnages connus ou jugés méritants. Par ailleurs, par opposition à une ville comme Montréal par exemple, on évite les noms d'axes routiers dont la longueur rendrait impossible la localisation de la station dans l'espace urbain. Enfin, selon les périodes, on privilégie (ou non) dans la mesure du possible des noms unilingues (Merode, Tomberg, Hankar, Diamant, Beekkant, etc.). À défaut, on a souvent préféré des noms dont les orthographes française et néerlandaise sont proches (Parc / Park, Saint-Guidon / SintGuido, Osseghem / Ossegem, Louise / Louiza, etc.).

\section{Les noms de stations : entre poids des lieux, règles, valeurs et interférences politiques}

\section{Les noms qui s'imposent de fait}

Pour un certain nombre de stations, le choix du nom n'a posé aucune question. Il en va ainsi des gares (du Midi, Centrale, du Nord, etc.) et pour différents lieux emblématiques comme la Bourse et certaines portes (de Namur, de Hal). Le nom de station correspond également bien à divers lieux suffisamment connus à l'échelle suprarégionale - (le plateau du) Heysel, (jardin et centre culturel du) Botanique, etc. - ou régionale - (place) Bockstael, Parvis de Saint-Gilles, (campus du) CERIA, etc. Ailleurs, dans de nombreux cas, une place, le nom du quartier traversé ou un équipement collectif ont permis de trouver aisément un nom de station: (place) Bizet, (place des) Étangs Noirs, (quartier) Beekkant, (quartier) Stockel, (hôpital) Érasme, (quartier et cité-jardin) La Roue, (quartier) Tomberg, etc. Dans un seul cas, on a appliqué la formule parisienne du nom composé de deux axes se croisant au niveau de la station : Arts-Loi correspond ainsi à la 
même logique que Réaumur-Sébastopol, Barbès-Rochechouart ou Sèvres-Babylone ${ }^{7}$. Dans tous ces cas, le nom de la station est clairement une aide au repérage, s'appuyant sur un nom de lieux préexistant et connu du plus grand nombre, voire étant logique pour des usagers n'étant pas familiers de la ville, sous réserve de maitrise du français ou du néerlandais.

Parfois, le nom de la station aide à avaliser dans l'inconscient et la pratique collectifs une évolution urbaine majeure. Ainsi, la station Rogier rappelle aux éventuels distraits que la gare du Nord ne s'y trouve plus, et renforce l'identité toponymique du lieu bien plus fortement sans doute que la médiocrité de l'espace public local, hérité des grands travaux routiers des Golden Sixties. À une échelle plus locale, la station de métro Gare de l'Ouest acte la translation de la gare ferroviaire homonyme de plusieurs centaines de mètres vers le sud.

\section{Quand un lieu mineur ou un nom peu connu deviennent des marqueurs urbains}

Cependant, l'application des règles ou guides évoqués plus haut, et en particulier l'évitement de noms de longs axes ${ }^{8}$ et la recherche de noms courts et si possible unilingues, va mettre de côté un certain nombre de noms de lieux qui auraient pu passer pour emblématiques (portes d'Anvers, de Laeken, de Schaerbeek, de Louvain, etc.) tout en faisant émerger des noms de lieux mineurs d'un point de vue urbanistique et/ou de la renommée.

Ainsi, Botanique / Kruidtuin a été préféré à Porte de Schaerbeek / Schaarbeeksepoort. Dans la mesure où l'on y trouve le parc et le centre culturel du même nom, cela ne s'avère guère gênant. Tout au plus certains regretteront la disparition, dans la connaissance du public, d'un nom d'ancienne porte. À l'est du parc du Cinquantenaire, Merode plutôt que Porte de Tervueren / Tervurensepoort (ou Parc du Cinquantenaire / Jubelpark) est un modèle d'efficacité. Il permet aussi de mettre à l'honneur la maison princière de Merode, qui fait partie de la haute aristocratie belge ainsi que de l'historiographie nationale. Cependant, le square Princesse Jean de Merode est un lieu en retrait et tout à fait marginal par rapport à la composition monumentale de l'avenue de Tervueren. Très peu d'utilisateurs de la station savent sans doute où se trouve ledit square. Il n'empêche que le nom s'est sans conteste imposé, illustrant le fait que le nom d'une importante station de métro peut donc prendre le dessus sur un lieu localement mineur. "Rendez-vous à Merode" entend-on dire aujourd'hui à l'occasion d'un appel par téléphone portable.

Si Merode montre bien la mise en exergue d'un lieu mineur cependant associé à une illustre famille, dans de nombreux autres cas, c'est un lieu mineur associé à un nom mineur qui a été choisi pour parvenir à l'efficacité de la communication. Ainsi, la station Pétillon renvoie à la rue Major Pétillon, artère locale parallèle à la ligne, plutôt qu'à l'avenue des Volontaires, plus importante mais fort longue et imposant une traduction en Vrijwilligers. Ici aussi, le nom retenu est simple et efficace et finit par s'imposer au plus grand nombre, même si peu de gens connaissent le major, officier de l'État indépendant du Congo et conseiller communal d'Etterbeek où il mourut en 1909. Dans la même veine, on peut signaler la station Demey (rue locale dédiée à un bourgmestre de l'entre deuxguerres), faute d'autre nom s'imposant. Ou la station Thieffry, qui renvoie à une rue ne donnant pas directement accès à la station, mais offre à nouveau un nom court et unilingue. Qui plus est, il fait honneur à l'aviateur connu pour ses exploits durant la 
Première Guerre mondiale puis entre la Belgique et le Congo qu'il fut le premier à relier par avion, en 1925. Comme nous l'a aimablement signalé un des directeurs consultés pour cette recherche, l'aviateur est ainsi passé de l'air au sous-sol...

Dans certains cas, l'impossibilité de trouver un nom répondant aux guides officieux impose des noms inconnus et le contournement desdites recommandations. Ainsi, Herrmann-Debroux, abrégé en H.-Debroux ${ }^{9}$ pour tenir sur les afficheurs électroniques et issu de l'avenue du même nom, fut choisi pour le terminus faute d'autre nom porteur d'une quelconque identité en dehors du boulevard du Souverain beaucoup trop long (plus de $4 \mathrm{~km}$ ). En outre, on pensait alors que le métro serait prolongé jusqu'au quartier du Transvaal, qui aurait donné un terminus simple, court et unilingue. Mais à nouveau, le nom s'est imposé et fait aujourd'hui partie de l'espace vécu d'un grand nombre de personnes, même non utilisatrices des transports collectifs.

\section{Les valeurs des élites, mythes, clivages et symboles nationaux}

19 Par opposition à certaines villes d'autres pays, la toponymie des rues bruxelloises ne se caractérise pas particulièrement par l'exaltation incessante du pouvoir en place, de l'unité nationale, de la colonisation passée et des héros de la révolution ou d'illustres batailles militaires. Ceux-ci ont certes droit à leur rue ou place, mais en quantité limitée. Cela n'a cependant pas empêché de mettre en avant quelques personnes perçues comme illustres ou héroïques, certes dans le cadre des règles officieuses présentées plus haut (Thieffry, Merode).

20 La famille royale a eu les honneurs du métro, quoique assez tardivement finalement. Passons rapidement sur Joséphine-Charlotte, au square du même nom. Princesse de Belgique puis grande-duchesse de Luxembourg, elle n'est pas la figure royale la plus connue ou la plus emblématique de la Belgique. La station Stuyvenbergh fait écho à la rue, au parc et au château du même nom malgré leur relatif éloignement. Il est en concordance avec la décoration de la station, centrée sur la reine Élisabeth qui vivait au château de Stuyvenbergh. Mais c'est surtout le décès du roi Baudouin en 1993 qui fit plus visiblement entrer la royauté dans le métro. D'une part, la même année, la station Luxembourg est rebaptisée à l'initiative du ministre régional des transports, socialchrétien. Elle s'appellera désormais Trône, ce qui n'est pas illogique vu qu'elle se situe en bordure de la place éponyme. D'autre part, le terminus nord-occidental du métro, inauguré en 1998, devait initialement s'appeler Amandiers / Amandelbomen, nom fort long qui aurait posé des problèmes d'affichage. Dans la foulée de la mort du roi, la station est finalement baptisée Roi Baudouin/ Koning Boudewijn ${ }^{10}$, le stade du Heysel tout proche ayant été préalablement lui aussi ainsi renommé. Faut-il y voir une conséquence de l'ampleur, du moins côté francophone, de l'émotion populaire suscitée par le décès inattendu et de son relais par une presse francophone jouant dans le registre de l'émotion et de la mémoire plutôt que de l'analyse et de la critique (van Ypersele, 1998) ? Toujours est-il que la royauté n'est donc mise à l'honneur que tardivement de manière explicite ${ }^{11}$, alors que l'État, bientôt officiellement fédéral, est affaibli par la régionalisation / communautarisation du pays. A tort ou à raison, le Roi Baudouin et la famille royale ont cependant passé pour le ciment de l'unité du pays. Quant au roi Léopold II et à la princesse Elisabeth, leur nom n'est apparu dans le métro qu'à l'occasion du récent "bouclage" de la ligne 2/6. Celle-ci passant deux fois à la station Simonis mais à des niveaux différents, l'exploitant a différencié ces derniers par les mentions "Simonis 
(Leopold II)", en néerlandais sans traduction, et "Simonis (Elisabeth)", en français sans traduction ${ }^{12}$.

Quelques autres grandes figures plus récentes de l'histoire ou de l'historiographie belges ont également donné leur nom à une station, mais au prix d'une rupture totale avec la toponymie locale. On reviendra plus loin sur Eddy Merckx. Il y a bien sûr Jacques Brel. Si le nom ne trouve pas d'écho dans la toponymie du quartier desservi, celui-ci est cependant imprégné du célèbre chanteur puisqu'il connut le tram $33^{13}$ et la cartonnerie familiale. Le célèbre architecte de l'Art Nouveau, Victor Horta, donne quant à lui son nom à une nouvelle station en 1993, en période de remise à l'honneur de son courant architectural mais en rupture avec la toponymie locale qui aurait logiquement conduit à préférer "Barrière" (de Saint-Gilles), tandis que le musée Horta est situé à plus de 800 mètres. Il est vrai cependant que la station est dotée de ferronneries issues de la Maison du Peuple et de vitraux de l'Hôtel Aubecq.

Étonnamment, ni Tintin ni son auteur Hergé n'ont officiellement donné nom à une station. Est-ce à cause d'un passé pour le moins discutable durant la Seconde Guerre mondiale ? Ceci ne les a pourtant pas empêchés d'accéder au rang de mythe belge, en fait comme mythe de remplacement pour un peuple (ou partie de peuple?) nostalgique d'un pays jadis supposé plus uni, ambitieux, colonialiste et entreprenant, tel qu'on le retrouve dans les premiers albums de Tintin (Kotek, 1995). Hergé en est devenu intouchable. Pourtant donc, point de station nommée Tintin ou Hergé. Ceux-ci sont cependant apparus "par la bande" à la station Stockel. À son ouverture en 1988, celle-ci est une longue fresque représentant plus de 140 personnages issus des aventures de Tintin, dessinés par l'auteur juste avant sa mort. Puis, en 2009, dans la station, on ajouta la signature personnelle de Hergé à côté de la mention "Stockel". Cette signature n'apparaît cependant pas sur les plans et autres éléments de signalétique de l'exploitant. Est-ce trop coûteux ou y a-t-il une certaine gêne?

On observe également une valorisation différenciée des métiers. On célèbre ainsi l'Hôtel des Monnaies, où des monnaies belges et étrangères étaient frappées, même si celui-ci était éloigné de quelques centaines de mètres par rapport à la station cependant située à une extrémité de la longue rue Hôtel des Monnaies. Mais de moins nobles métiers n'ont guère eu droit à pareil honneur. Ainsi, l'ancien marché aux poissons, disparu mais jusqu'à ce jour connu sous le nom de vismet, est desservi par une station Sainte-Catherine, alors que la place Sainte-Catherine se trouve un peu plus loin. Trop populaire, l'ancien marché était peut-être aussi trop lié au vieux Bruxelles que l'on transformait alors physiquement et socialement à coups de bulldozers (Demey, 1992; Dessouroux, 2008). Par ailleurs, la station qui dessert les Abattoirs ne s'appelle pas ainsi, ce qui, il est vrai, aurait imposé une traduction (Slachthuizen). Comme la station fut un terminus durant plus d'une décennie, on a sans doute voulu éviter que les passagers prennent le métro pour l'abattoir. Mais en matière de boucherie, nommer la station Clemenceau, du nom d'une rue proche de la station, peut laisser perplexe vu ses positions relatives à la Première Guerre mondiale ${ }^{14}$. Enfin, la station desservant le quartier jadis industriel de la rue de Manchester devait porter son nom, le quartier étant d'ailleurs connu comme étant le "petit Manchester". Las, elle fut finalement baptisée Delacroix, du nom d'une rue proche, tournant ainsi le dos au passé industriel. A-t-on préféré faire honneur à celui qui vit, en tant que premier ministre des années 1918-1920, l'instauration du suffrage dit universel (en fait pour les seuls hommes) ? Car dans ce cas, le message eût été plus clair si la décoration de la station avait rendu hommage aux libertés démocratiques... 
24 Le métro de Bruxelles a également permis l'expression du clivage entre laïques et catholiques, constitutif de la vie sociale et politique belge depuis le XIX ${ }^{\mathrm{e}}$ siècle, même s'il est aujourd'hui à bien des égards dépassionné (Marissal et al., 2007). La desserte des campus de l'Université libre[-exaministe] (ULB, laïque) et de l'Université catholique de Louvain (UCL) fut en effet l'occasion d'expression de ce clivage, quoique de manière discrète. Le campus de la Plaine de l'ULB est desservi par une station dénommée Delta. Malgré les différentes interprétations entendues, il semblerait que ce nom fut choisi comme référence directe au nouveau campus de l'Université libre de Bruxelles dont le triangle d'origine maçonnique est un symbole de premier plan pour une université dont les fondateurs affirmaient le refus de tout dogme ("Scientia Vincere Tenebras") (Unger et al., 2004) ${ }^{15}$. L'impersonnel carrefour sis au-dessus de la station est en tout cas un bel exemple de "non-lieu" devenu un nom de lieu connu du plus grand nombre, jusque dans le radioguidage destiné aux automobilistes.

La Belgique ne serait pas ce qu'elle est sans une anecdote similaire pour la station desservant l'implantation bruxelloise de l'Université catholique de Louvain (UCL), nommée Alma. Ce nom est une simplification de l'Alma Mater et rappelle l'Alma de l'université de Leuven, d'où les francophones furent chassés en 1968 (crise du "Walen buiten"16). À Leuven, l'Alma est en effet le lieu central de sociabilité et de services à la communauté universitaire. En donner le nom à la station de métro aurait été une demande du Baron Fallon, alors bourgmestre catholique de Woluwe-Saint-Lambert.

Enfin, le métro n'échappe parfois pas au fait du prince. Une nouvelle station à Anderlecht devait porter le nom du poète belge Maurice Carème, appellation qui tenait à coeur au ministre régional des transports malgré l'éloignement du boulevard du même nom. Las, le jour de l'inauguration le ministre avait changé et le nouveau avait entre-temps imposé le mythique coureur cycliste Eddy Merckx, un proche du nouveau ministre. L'exploitant tenta de s'y opposer, non à cause de la rupture avec la toponymie locale mais parce que le cycliste devenait le premier à donner son nom à une station de son vivant, ouvrant potentiellement la porte à des dérives ou demandes en tout genre ${ }^{17}$. À l'heure de l'affaissement du rôle distributeur de la puissance publique, du contrôle des chômeurs et de "l'activation" des demandeurs d'emploi, on peut en outre se demander si le choix d'un personnage symbolisant la réussite par l'effort individuel ne s'inscrit pas, certes inconsciemment, dans les créneaux idéologiques du moment.

\section{Des enjeux linguistiques?}

Enfin, il eût été étonnant que le clivage linguistique qui traverse la Belgique et Bruxelles ne finisse pas par atteindre le métro bruxellois.

De manière générale, les stations, fussent-elles de nom bilingue, ont en quelque sorte une identité linguistique. Dans la communication écrite (notamment sur les plans) et orale (annonces dans les rames), une station donnée sera en effet d'abord présentée en français puis en néerlandais, et inversement pour la suivante. Et si un nom est unilingue, le message sonore annonçant la station sera émis avec la voix et l'accent de la langue concernée... Ainsi, la Gare Centrale est d'abord "francophone" (Gare Centrale / Centraal Station). Vers l'est, vient ensuite Park / Parc, qui sera vocalement annoncée Park avec une voix flamande, puis Arts-Loi / Kunst-Wet, Maalbeek / Maelbeek (voix flamande), Schuman (voix francophone), etc. L'alternance linguistique est donc la règle. 

était Bornival, du nom d'une petite rue débouchant en face de la station. Ce nom fut donc abandonné à la suite d'un choix qui peut paraître déroutant puisqu'il renvoie à la commune flamande sise 350 mètres plus loin, au-delà de la limite de la Région de Bruxelles-Capitale. Voilà donc l'exploitant des transports collectifs bruxellois disposant d'une station portant le nom d'une commune de la Région flamande. Faut-il y voir une manoeuvre "flamande" visant à faire symboliquement entrer la Flandre dans Bruxelles ${ }^{18}$ ? À l'inverse, faut-il plutôt y voir une manoeuvre "francophone" de lier à Bruxelles une commune flamande en fait largement francisée du fait de la péri-urbanisation des francophones bruxellois? Nous n'avons hélas pu trancher la question.

\section{Discussion et conclusions}

À Bruxelles, la fonction toponymique principale des stations de métro est clairement l'aide au repérage pour une infrastructure créée après urbanisation. Par opposition à d'autres nécessités ou volontés contemporaines de nommer des lieux ou espaces, on n'est donc pas ici dans le jeu dialectique entre idéologie territoriale et pouvoir politique ainsi légitimé (voir les notions de "pays" local, de nouvelles régions, d'intercommunalités, etc.) (Di Méo et Anglade, 1996). On n'est pas non plus dans une logique de ville nouvelle où l'on doit inventer des noms pour tenter de forger une identité aux lieux à défaut d'histoire urbaine. La fonction d'aide au repérage dans la ville contribue donc fortement à rendre apolitique et sans véritables enjeux le choix du nom des stations.

Ceci n'empêche cependant pas l'existence de filtres sociaux et politiques, éventuellement inconscients, qui conduisent à valoriser ou, au contraire, à écarter tel ou tel nom potentiel. Ainsi en va-t-il des personnages historiques, des héros populaires ou des métiers. Outre le clivage social, on peut également trouver trace, certes marginalement, des clivages philosophique et linguistique, autres piliers traditionnels de la Belgique (Marissal et al., 2007).

Figure 2. Liens entre les stations de métro et leur quartier.

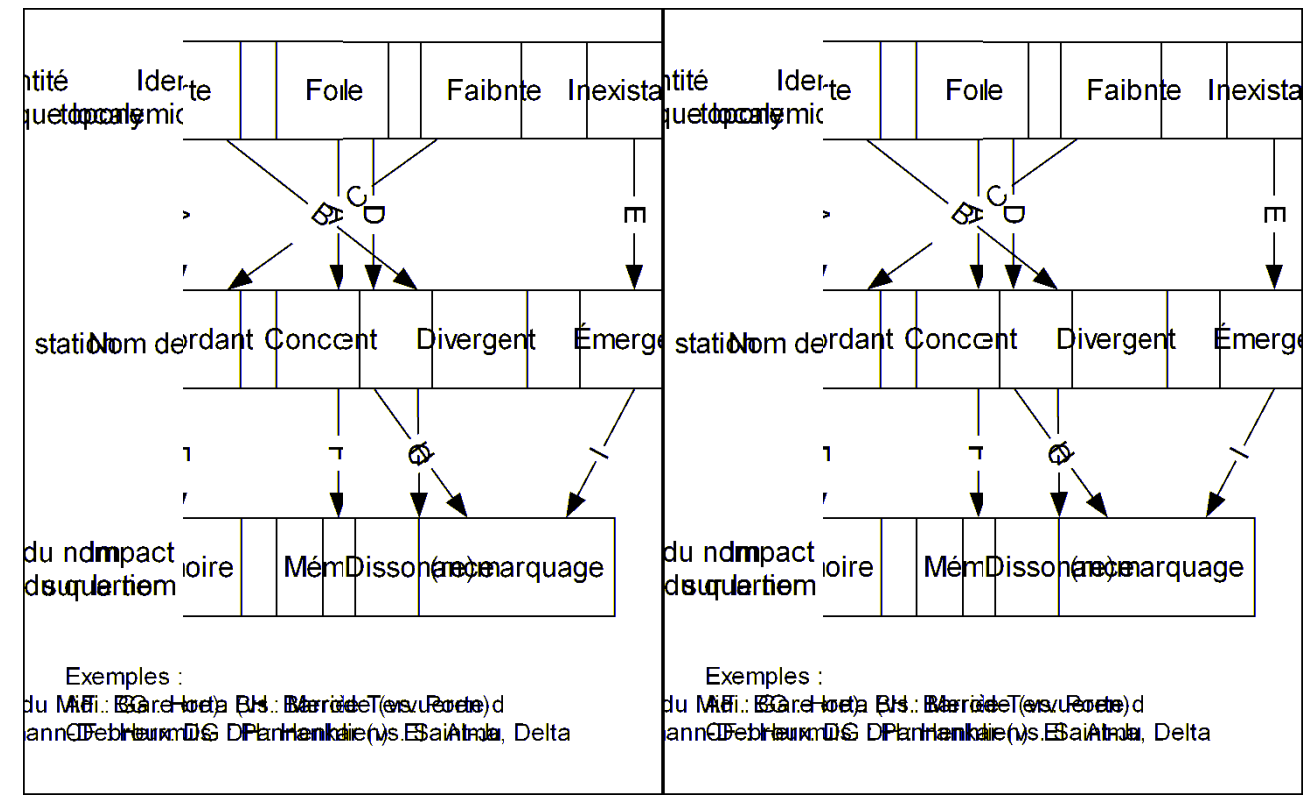

Belgeo, 1-2 | 2010 
$32 \mathrm{Au}$ travers des exemples présentés, on se rend compte qu'il existe cependant une diversité de rapports entre la toponymie locale, le nom des stations et l'impact de ces derniers (mémoire vs. (re)marquage) (figure 2). Toutefois, ce qui frappe peut-être le plus avec le métro bruxellois, c'est l'émergence d'une galerie de lieux géographiquement secondaires dans l'espace urbain (par élimination, rappelons-le, de noms des longs axes) et, partant, de noms souvent peu ou pas connus. De ce fait, le nom des stations de métro y officie plus souvent qu'ailleurs comme marqueur urbain (par émergence de noms préalablement connus des seuls initiés du quartier concerné) - ou éventuellement comme re-marqueur si le nom de la station tranche avec la toponymie locale - plutôt que comme fonction mémorielle de lieux déjà connus. Quoi qu'il en soit, les noms s'imposent aux usagers de l'infrastructure, voire à un public plus large. C'est ainsi qu'à la Révolution, aux supposés grands généraux, aux présidents presque déifiés et aux mythiques batailles que l'on retrouve dans les rues et stations du métro parisien, celui de Bruxelles offre des noms d'apparence neutre et généralement éloignés de l'historiographie, à l'exception sans doute d'Eddy Merckx, Hergé ("par la bande") et le Roi Baudouin après sa mort. Finalement, le nom des stations du métro bruxellois n'est-il pas à l'image de la Belgique : d'apparence consensuelle et peu pourvu en mythes fondateurs?

\section{Remerciements}

Cette courte recherche n'aurait pu être menée à bien sans l'aide précieuse de MM. J.-P. Alvin, Ch. Dochy, C. van den Hove, G. Verheulpen et M. Woitchik, que je remercie chaleureusement pour le temps consacré et l'effort de mémoire consenti. Merci également à Mathieu Van Criekingen (ULBIGEAT) pour ses commentaires critiques.

\section{BIBLIOGRAPHIE}

BASSE P.-L. (2007), Guy Môquet au Fouquet's, Paris, Éd. des Equateurs, 46 p.

BERG L., VUOLTEENAHO J. (2009) (eds), Critical Toponymies. The Contested Politics of Place Naming, Ashgate, $306 \mathrm{p}$.

BROCCOLINI A. (2001), “Toponymie et identité à Naples : la napolitanité du quartier Santa Lucia (XIX et XX $\mathrm{X}^{\mathrm{e}}$ siècles), in BOUVIER ET GUILLON, La toponymie urbaine. Significations et enjeux, pp. 171-187.

BOUVIER J.-C., GUILLON J.-M. (2001)," Introduction “de La toponymie urbaine. Significations et enjeux , pp. 103-110.

CLEMENCEAU G. (1934), Discours de guerre, publiés par la Société des amis de Clemenceau, Paris, Plon, $300 \mathrm{p}$.

DEMEY T. (1992), Bruxelles, chronique d'une capitale en chantier, tome 2 : De l'Expo '58 au siège de la C.E.E., Bruxelles, Ed. Paul Legrain, 329 p.

DESSOUROUX C. (2008), Espaces partagés, espaces disputés. Bruxelles, une capitale et ses habitants, Bruxelles, MRBC. 
DI MÉO G., ANGLADE M. (1996)," Identité, idéologie et symboles territoriaux. L'exemple du VicBilh en Béarn “, in DI MÉO G. (dir.), Les territoires au quotidien, Paris, L’Harmattan, pp. 87-110.

DI MÉO G., BULÉON P. (dir.) (2005), L'espace social : une lecture géographique des sociétés, Paris, Armand Colin, $303 \mathrm{p}$.

GIRAUT F., HOUSSAY-HOLZSCHUCH M., GUYOT S. (2008)," Au nom des territoires ! Enjeux géographiques de la toponymie “, L’Espace Géographique 37, 2008-2, pp. 97-105.

GUILLOREL H. (dir.) (2009), Toponymie et politique. Les marqueurs linguistiques du territoire, Bruxelles, Bruylant, $234 \mathrm{p}$.

JAUMAIN S. et al. (2011), Dictionnaire de Bruxelles, Bruxelles, Le Cri (à paraître).

KOTEK J. (1995)," Tintin, un mythe belge de remplacement “, in MORELLI A. (dir.), Les grands mythes de l'histoire de Belgique, de Flandre et de Wallonie, Bruxelles, Édition Vie Ouvrière, pp. 281-292.

LAPORTE C. (1999), L'affaire de Louvain, 1960-1968, Bruxelles, De Boeck Université, 333 p.

MARISSAL P., MEDINA LOCKHART P., VAN HAMME G., VANDERMOTTEN C. (2007), Atlas de

Belgique, tome 1 : Géographie politique, Gent, Academia Press, 82 p.

MICHONNEAU S. (2001),” Nouvelle toponymie, nouvelle identité. Le cas de la Barcelone des agrégations (1897-1910) “, in BOUVIER et GUILLON, La toponymie urbaine. Significations et enjeux, pp. 151-169.

MUSSET A. (à paraître)," Le plan du métro de Mexico : points, lignes et territoires “, Le monde des cartes, 204.

PINCHEVSKI A., TORGOVNIK E. (2002)," Signifying passages: the signs of change in Israeli street names “, Media Culture Society, 24, 3, pp. 365-388.

RICHARD É. (2001)," Femmes dans la rue ou les 'trous de mémoire' de la ville, l'exemple marseillais “, in BOUVIER et GUILLON, La toponymie urbaine. Significations et enjeux, pp. 103-110.

ROSE-REDWOOD, R., ALDERMAN, D., AND AZARYAHU, M. (à paraître)," Geographies of Toponymic Inscription: New Directions in Critical Place-Name Studies “, Progress in Human Geography.

UNGER J. et al. (2004), Itinéraire de l'Université Libre de Bruxelles, Bruxelles, Société Royale Belge de Géographie, coll. Hommes et Paysages, 80 p.

VAN YPERSELE L. (1998)," La nation dit adieu à son Roi “, in KONINCKX C. et LEFÈVRE P. (dir.) Le roi Baudouin : une vie, une époque, Bruxelles, Racine, pp. 245-257.

VUOLTEENAHO J., BERG L. (2009)," Towards Critical Toponymies ", in BERG L., VUOLTEENAHO J. (eds.), Critical Toponymies. The Contested Politics of Place Naming, Ashgate, pp. 1-18.

\section{NOTES}

1. Au sens premier du terme, c'est-à-dire s'opposant au progressisme.

2. Calculs et constats personnels d'après Google Maps.

3. Pour une description systématique de l'origine du nom des stations du métro bruxellois, voir les notices correspondantes dans Jaumain S. et al. (2011), à paraître.

4. Numéro 2008-2, disponible sur http://espacepolitique.revues.org/index124.html

5. Nous les en remercions très chaleureusement.

6. Instituée par Arrêté Royal du 7 avril 1926 et travaillant sous le haut patronage de l'Académie royale des Sciences, des Lettres et des Beaux-Arts de Belgique et de la Koninklijke Vlaamse 
Academie van België voor Wetenschappen en Kunsten, cette commission "a pour but principal de développer et d'encourager les études de toponymie et de dialectologie". Cependant, en plus de cet objectif scientifique originel, la Commission s'est rapidement penchée sur la question de l'orthographe du nom des lieux (communes, lieux apparaissant sur les cartes d'état-major, etc.). Dans ce cadre, elle se tient à la disposition des pouvoirs publics à titre consultatif. Voir http:// www.toponymie-dialectologie.be.

7. Une variante étant cependant la station Houba-Brugmann, contraction faisant référence à l'avenue Houba de Strooper et à l'hôpital Brugmann plus ou moins proche.

8. L'exception notoire étant la station Roodebeek, à l'extrémité d'une chaussée de Roodebeek de deux kilomètres de long et prolongée, à l'autre extrémité, par une avenue de Roodebeek d'un kilomètre de long.

9. Le trait d'union est souvent omis, laissant penser, à tort, que le H. renverrait au prénom.

10. Abrégé en Baudouin / Boudewijn sur les afficheurs électroniques.

11. Joséphine-Charlotte tient vraisemblablement plus du hasard de la toponymie locale et Stuyvenbergh de la difficulté à trouver un autre nom. De plus, il n'est pas certain que le public fasse spontanément le lien entre la famille royale et Stuyvenbergh, si ce n'est par la décoration de la station.

12. L'absence de traduction (Léopold avec accent en français et Elizabeth avec un $Z$ en néerlandais) provient d'une contrainte au niveau des afficheurs électroniques, prévus pour faire alterner maximum deux mentions. La longueur des nouveaux noms implique un affichage alterné (par exemple "Simonis" en alternance avec "Elisabeth", rejetant de facto une mention dans l'autre langue). Cela étant, s'agissant de prénoms de personnes, l'usage voudrait normalement que ceux-ci demeurent dans la langue des intéressés, donc "Léopold II" avec accent et "Elisabeth" avec un "s".

13. "On prendra le tram 33, Pour manger des frites chez Eugène" (Madeleine, 1962).

14. Comme parlementaire puis comme président du Conseil et ministre de la Guerre à partir de 1917, Clemenceau n'a cessé de défendre l'idée que la "civilisation française" devait vaincre la "barbarie germanique". Opposé à toute idée de paix négociée (la paix ne pourrait donc venir que de la victoire), il fut intraitable avec les "traitres" et les "anti-patriotiques". Voir Clemenceau (1934).

15. Les autres interprétations renvoient aux proches voies de chemin de fer formant un triangle ou au dessin que forment les voies de métro se déployant vers l'atelier-dépôt adjacent, mais il semble qu'il s'agisse d'interprétations ex post.

16. "Les Wallons dehors". Notons cependant que la décision d'implanter à Bruxelles la section francophone de la Faculté de Médecine est antérieure à la crise ayant conduit à la scission (Laporte, 1999).

17. Pour de futures stations, d'imaginatifs fonctionnaires m'ont à ce propos suggéré, non sans humour, le Grand Jojo et Annie Cordy.

18. Rappelons que par fusion de la Région flamande et de la Communauté flamande, une certaine Flandre présente ou revendique Bruxelles comme partie intégrante de la Flandre. 


\section{RÉSUMÉS}

Cet article s'intéresse au nom donné par les pouvoirs publics aux stations du métro de Bruxelles. Alors que donner un nom aux lieux est un acte pouvant correspondre à des choix politiques et idéologiques, baptiser les stations de métro suppose a priori d'aider les voyageurs à se repérer par rapport à un tissu urbain généralement préexistant à l'infrastructure de transport. Dans un certain nombre de cas, l'identité toponymique des lieux s'impose en effet à la station. Dans d'autres cas, donner un nom aux stations a cependant impliqué des choix et l'expression de valeurs conscientes ou inconscientes, dont témoignent par exemple la mise en valeur différenciée des métiers et des personnages, des options linguistiquement connotées et divers écarts aux "règles". Finalement, le nom des stations peut conforter les noms des lieux, conduire à un re-marquage de ceux-ci ou demeurer dissonant.

This article looks into the names given by public authorities to underground stations in Brussels. If naming places can reflect political and ideological choices, naming underground stations supposes first of all helping travellers find their way within a - generally - preexisting urban fabric.

In some cases, the toponimic identity of places is obvious, whereas naming stations is sometimes the expression of choices and of conscious or unconscious values, as shown by the differentiated celebration of crafts or public figures, linguistically connoted options, as well as diverse deviations from the "rules". Finally, the names of stations can consolidate names of places, remark them, or remain dissonant.

\section{INDEX}

Mots-clés : Bruxelles, métro, toponymie, néotoponymie, toponymie urbaine Keywords : Brussels, underground, toponymy, place naming, critical toponymy, urban toponymy

\section{AUTEUR}

\section{FRÉDÉRIC DOBRUSZKES}

Université Libre de Bruxelles (IGEAT), frederic.dobruszkes@ulb.ac.be 